\title{
Experimental investigation of the kinetic energy correction factor in pipe flow
}

\author{
Tomasz Janusz Teleszewski ${ }^{1, *}$ \\ ${ }^{1}$ Department of HVAC Engineering, Faculty of Civil and Environmental Engineering, Bialystok \\ University of Technology, Wiejska 45A, 15-351 Bialystok, Poland
}

\begin{abstract}
In this study, an experimental investigation of the kinetic energy (Coriolis) correction factor in laminar, transitional and turbulent flow in a transparent smooth pipe with a Reynolds number up to 25000 is performed. The velocity profiles are obtained using a laser Doppler velocimetry (LDV). Based on the experimental results obtained for $\operatorname{Re}<25000$, generalized correlations for the kinetic energy correction factor as a function of the Reynolds number are presented. The results of the research are compared with experimental results reported by other authors. The predicted correlations for the kinetic energy correction factor can be a very useful resource for the hydraulic calculations of fluid through circular ducts.
\end{abstract}

\section{Introduction}

In many environmental engineering issues, it is necessary to calculate the hydraulic flow of fluid through circular ducts $[1,2]$. Flow in smooth pipes has been extensively investigated by many researchers [3-6]. In hydraulic calculations where the Bernoulli equation [3] is applied, the simplification of the mean velocity $\mathrm{u}_{1}$ and $\mathrm{u}_{2}$ is introduced:

$$
\frac{u_{1}^{2}}{2 g}+\frac{p_{1}}{\rho g}+z_{1}=\frac{u_{2}^{2}}{2 g}+\frac{p_{2}}{\rho g}+z_{2}+\Delta h
$$

where: $p_{1}$ and $p_{2}$ are the pressures in sections 1 and $2, z_{1}$ and $z_{2}$ are the height above reference level in sections 1 and $2, g$ is the acceleration due to gravity, $\rho$ is the density and $\Delta h$ is hydraulic losses.

The mean velocity $u_{m}$ can be determined from the following relationship [7]:

$$
u_{m}=\frac{Q}{A}
$$

where: $Q$ is the volume flow rate and $A$ is the cross-sectional area of the duct.

\footnotetext{
*Corresponding author: t.teleszewski@pb.edu.pl
} 
The shear stresses in the fluids during flow cause a nonuniform distribution of velocities in the cross-sections. The use of an average velocity in the form of a uniform distribution results in errors in the kinetic energy component. Therefore, a Coriolis correction factor $\alpha$ $[1,2,7]$ was introduced in order to perform accurate hydraulic calculations in the Bernoulli equation (1):

$$
\frac{\alpha_{1} u_{1}^{2}}{2 g}+\frac{p_{1}}{\rho g}+z_{1}=\frac{\alpha_{2} u_{2}^{2}}{2 g}+\frac{p_{2}}{\rho g}+z_{2}+\Delta h
$$

The ratio of the actual stream of kinetic energy to a stream computed from average speed, which is a Coriolis factor, is determined from the following formula [7]:

$$
\alpha=A \frac{\int_{A} u^{3} d A}{A u_{m}^{3}}
$$

where: $u$ is the velocity at each point of the cross section.

The kinetic-energy correction factor $\alpha$ may be determined for laminar flow in a tube by use of the well-known parabolic Hagen-Poiseuille velocity profile for fully developed laminar flow:

$$
u=u_{c}\left(1-\left(\frac{r}{R}\right)^{2}\right)
$$

where $u_{c}$ is a centerline velocity, $r$ is a radial coordinate and $R$ is the radius of a pipe. The mean velocity is defined by (2) and gives, for laminar flow $\mathrm{u}_{\mathrm{m}}=0.5 \mathrm{u}_{\mathrm{c}}$. Using Eqn. (5) in Eqn. (4), the kinetic-energy correction factor is equal to 2 [7]. For turbulent flow the velocity distribution in the main stream can be represented by the relation $[8,9]$ :

$$
u=u_{c}\left(1-\frac{r}{R}\right)^{m}
$$

where $m$ is independent of the Reynolds number. Substituting Eq. (6) into (4) the kinetic energy correction for turbulent flow is defined as

$$
\alpha=\frac{(1+m)^{3}(2+m)^{3}}{4(1+3 m)(2+3 m)}
$$

In Table 1, some numerical values $\alpha$ are reported [7].

Table 1. Kinetic energy correction factor for some values of the coefficient $\mathrm{m}$ for a pipe [7].

\begin{tabular}{|c|c|c|c|c|c|}
\hline $\boldsymbol{m}$ & $\mathbf{1 / 5}$ & $\mathbf{1 / 6}$ & $\mathbf{1 / 7}$ & $\mathbf{1 / 8}$ & $\mathbf{1 / 9}$ \\
\hline$\alpha$ & 1.106 & 1.077 & 1.058. & 1.046 & 1.037 \\
\hline
\end{tabular}

For turbulent flow with a Reynolds number between 5000 and 35000000, an empirical formula $\alpha(\operatorname{Re})$ has been suggested by Szewczyk [10]:

$$
\alpha=1+101\left(\frac{10}{\ln ^{2} \operatorname{Re}}\right)^{3}-10.7\left(\frac{10}{\ln ^{2} \operatorname{Re}}\right)+\frac{11.3}{\ln ^{2} \operatorname{Re}}
$$


Work by Strzelecka and Jeżowiecka-Kabsch [11] resulted in the following empirical relation for transitional and turbulent pipe flow $(\operatorname{Re}=2800-100000)$ :

$$
\alpha=1+105\left(\frac{10}{\ln ^{2} \operatorname{Re}}\right)^{3}-11.88\left(\frac{10}{\ln ^{2} \operatorname{Re}}\right)+1.208\left(\frac{10}{\ln ^{2} \operatorname{Re}}\right)
$$

The aim of this study is analysis of the kinetic energy correction factor on the laminar, transitional and turbulent flow in a tube subject to Reynolds number up to 25000 . No solutions were found for the kinetic energy correction factor in the laminar, transitional and turbulent flow. The influence of the Coriolis on the Reynolds number for values of Re up to 25000 of a circular duct is experimentally investigated. The velocity profiles for laminar, transitional and turbulent flow in a pipe are obtained using a laser Doppler velocimeter (LDV). Molki at al. [12] presented a brief historical overview of flow measurement techniques, with a particular emphasis on the laser Doppler velocimetry technique.

\section{Experimental apparatus and methodology}

The model of the research stand is schematically shown in Figure 1. It consists of two sub-systems: the flow delivery system with a circular pipe and the laser Doppler velocimeter (LDV) system. Investigated was a smooth transparent acrylic tube, $9000 \mathrm{~mm}$ long with an inner diameter $D$ of $30 \mathrm{~mm}$ and a wall thickness of $5 \mathrm{~mm}$. Flow from the centrifugal pump passed through reservoir 1, then through the circular duct with a flow conditioner, then through a rotameter with a control valve, and finally through reservoir 2 and the pump returning to reservoir 1 to begin the second cycle. The miniLDV sub-system consists of a transmitter and a receiver probe, a processing engine set and burst processor acquisition manager software manufactured by Measurement Science Enterprise, Inc. The LDV probe is characterized by the following parameters: the laser wavelength is equal to $658 \mathrm{~nm}$, the probe volume size is $100 \times 150 \mathrm{~nm}$ with fringe spacing of $4.93 \mu \mathrm{m}$, and velocity in the range of 0.001 to $1 \mathrm{~m} / \mathrm{s}$ was assessed by means of the LDV probe with a repeatability uncertainty of $0.1 \%$ and an accuracy of $99.7 \%$. The experimental error will be greater in the places near the wall, where velocities are lower. Each point of velocity was taken along the diameter of the circular duct by traversing in $0.25 \mathrm{~mm}$ steps, starting at $0.25 \mathrm{~mm}$ from the pipe wall and up to 0.25 from the adjacent wall. The processor acquisition manager software collected an average velocity of 100 signal bursts at each point. Munson et al. (10) provided the relationship for entrance length and diameter and Reynolds number for laminar and turbulent flow in circular pipe:

$$
\begin{array}{ll}
L_{E}=0.006 D \mathrm{Re}, & \operatorname{Re}<2000 \\
L_{E}=4.4 D \operatorname{Re}^{1 / 6}, & \operatorname{Re}>2000
\end{array}
$$

The distances between the inlet and the place where the velocity profiles were obtained is equal to $4500 \mathrm{~mm}$. In this study, fully developed flow is considered. At all times, the average temperature of the water in the tube was maintained at the level of $20.0^{\circ} \mathrm{C} \pm 0.3^{\circ} \mathrm{C}$. 


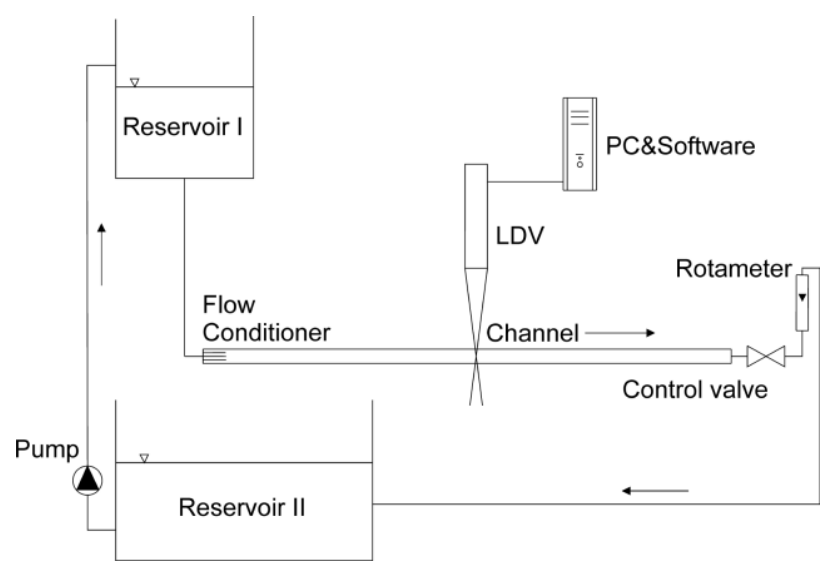

Fig. 1. Schematic of the experimental setup.

\section{Results and discussion}

Figure 2 is the graphical representation of the kinetic energy correction factor versus the Reynolds number ranging up to 25000. As the Reynolds number is increased, the variation of $\alpha$ with Re can be divided into several parts and points respectively. For laminar flow between the experimental results and the analytical solution $(\alpha=2)$ [7] with $\operatorname{Re}<2200$, a maximum deviations is equal to $1 \%$. The transition to turbulence flow was observed at a Reynolds number ranging from 2200 to 3000, and the kinetic energy correction factor rapidly decreased to 1.3 . For a Reynolds number from 3000 to 25000 the kinetic energy correction factor decreased from 1.3 to 1.1 . At the highest Reynolds number $(\operatorname{Re}>5000)$ studied, good agreement is observed between the obtained kinetic energy correction factor and the kinetic energy correction factor from literature $[10,11]$.

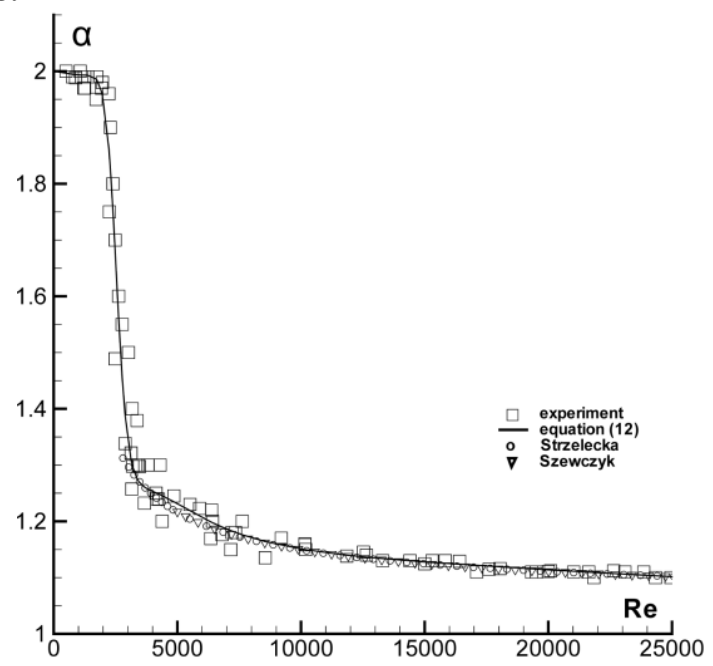

Fig. 2. Kinetic energy correction factor vs. Reynolds number $(\operatorname{Re}<25000)$ in pipe. Comparison of $\alpha(\mathrm{Re})$ formula with the data of literature [10,11].

The relationship for the kinetic energy correction factor and the Reynolds number ranging up to 25000 for a circular duct can be expressed in closed form as: 


$$
\alpha(\operatorname{Re})=\frac{2+a \mathrm{Re}^{0.5}+b \mathrm{Re}^{1}+c \mathrm{Re}^{1.5}+d \mathrm{Re}^{2}}{e+f \mathrm{Re}^{0.5}+g \mathrm{Re}^{1}+h \mathrm{Re}^{1.5}+i \mathrm{Re}^{2}+j \mathrm{Re}^{2.5}}
$$

where $a, b, c, d, e, f, g, h, i, j$ are reported in Table 2 .

Table 2. Coefficients of the Eq. (12) to determine $\alpha$ vs. Re.

\begin{tabular}{|c|c|c|c|c|}
\hline $\mathbf{a}$ & $\mathbf{b}$ & $\mathbf{c}$ & $\mathbf{d}$ & $\mathbf{e}$ \\
\hline-0.11945 & $2.758 \cdot 10^{-3}$ & $-3.01 \cdot 10^{-5}$ & $1.367 \cdot 10^{-7}$ & 1 \\
\hline $\mathbf{f}$ & $\mathbf{g}$ & $\mathbf{h}$ & $\mathbf{i}$ & $\mathbf{j}$ \\
\hline-0.0596 & $1.3647 \cdot 10^{-3}$ & $-1.43 \cdot 10^{-5}$ & $5 \cdot 10^{-8}$ & $1.66 \cdot 10^{-10}$ \\
\hline
\end{tabular}

Table 3 presents the results $\alpha$ from eq. 12 which are compared with analytical solution [7] for laminar flow and turbulent flow from literature [10,11]. Figure 2 also elucidates the kinetic energy correction factor versus the Reynolds number in the pipe from Eq. 12. The $\mathrm{R}^{2}$ determination coefficient is the measure of the model's fit. In the case of a relationship between the $\alpha$ and an increase in the Reynolds number in the pipe, it is equal to approx. 0.98 , which indicates a close relationship between the kinetic energy correction factor and an increase of Re (Fig. 2). For equation (12) with $\operatorname{Re} \in(5000 ; 25000)$, the maximum relative differences in the relation $\alpha=\mathrm{f}(\mathrm{Re})$ [10] and [11] were found to be $\Delta \alpha=1.2 \%$ and $\Delta \alpha=1.3 \%$, respectively. The uncertainty of the kinetic energy correction factor was calculated based on Eq. (4) using the method suggested by Moffat [13]. The uncertainty of the kinetic energy correction factor was estimated at approximately $1.4 \%$.

Table 3. Comparison between $\alpha$ versus Re obtained in this paper from eq. 12 with the results for circular duct found in literature.

\begin{tabular}{|c|c|c|c|c|}
\hline \multirow{2}{*}{$\operatorname{Re}$} & \multirow{2}{*}{$\boldsymbol{\alpha}$-present results (Eq. 12) } & \multicolumn{3}{|c|}{$\boldsymbol{\alpha}$-refs. [7, 10, 11] } \\
\cline { 3 - 5 } & & $\begin{array}{c}\text { analytical } \\
\text { solution }\end{array}$ & Szewczyk & Strzelecka \\
\hline 1000 & 1.99 & 2.00 & - & - \\
\hline 3000 & 1.34 & - & 1.30 & 1.30 \\
\hline 5000 & 1.23 & - & 1.22 & 1.22 \\
\hline 10000 & 1.15 & - & 1.15 & 1.15 \\
\hline 20000 & 1.11 & - & 1.11 & 1.11 \\
\hline
\end{tabular}

\section{Conclusions}

A laser Doppler velocimeter was used to measure the velocity profile for laminar, transitional and turbulent flow in a pipe for a Reynolds number ranging up to 25000. Mean velocity, Reynolds numbers and the kinetic energy correction factor were calculated from the LDV data. The experimental results of $\alpha$, shows good agreement with the results report by other authors for flows with a Reynolds number of $2800<\operatorname{Re}<25000$. The $\alpha=f(R e)$ dependence obtained in experimental studies is presented, with the aim of offering a very simple but accurate tool for engineers and designers involved in one-dimensional hydraulic applications.

The study has been implemented with the resources of the S/WBiIS statutory work financed by the Ministry of Science and Higher Education in Poland. 


\section{References}

1. C. Nalluri, M. Marriott, Civil Engineering Hydraulics (John Wiley and Sons, $5^{\text {th }}$ Edition, 2009)

2. A. Chadwick, J. Morfett, M. Borthwick, Hydraulics in Civil and Environmental Engineering (Spon Press, $5^{\text {th }}$ Edition, 2012)

3. D.D. Joseph, B.H. Yang, Physica D: Nonlinear Phenomena 239, 1318 (2010)

4. B.J. McKeon, M.V. Zagarola, A.J. Smits, J. Fluid Mech. 538, 429 (2005)

5. A. Ghanbari, F.F. Farshad, H.H. Rieke, Journal of Chemical Engineering and Materials Science 2, 6, 83 (2011)

6. S. Li, W. Huai, PLoS One 11, 5, e0154408 (2016)

7. F.M. White, Fluid Mechanics (McGraw-Hill, Boston, $4^{\text {th }}$ Edition)

8. L. Prandtl, O.G. Tietjens, Fundamentals of hydro- and aeromechanics (McGraw-Hill, New York, 1934)

9. von Karman, Aerodynamics (Cornell University Press, Ithaca, New York, 1954)

10. H. Szewczyk, Raporty Inst. Techn. Ciepl. i Mech. Płynów PWr, Seria PRE nr 7 (Wrocław, 2007)

11. K. Strzelecka, K. Jeżowiecka-Kabsch, Ochrona Środowiska 30, 1, 21 (2008)

12. A. Molki, L. Khezzar, A. Goharzadeh, Eur. J. Phys. 34, 1127 (2013)

13. R.J. Moffat, ASME, J. Fluids Eng. 107, 2, 173 (1985) 